\title{
Patient and caregiver characteristics associated with caregiver burden in Parkinson's disease: a palliative care approach
}

\author{
Zachary A. Macchi ${ }^{1 \#}$, Claire E. Koljack ${ }^{1 \#}$, Janis M. Miyasaki ${ }^{2}$, Maya Katz ${ }^{3}$, Nick Galifianakis ${ }^{3}$, \\ Lindsay P. Prizer ${ }^{4}$, Stefan H. Sillau ${ }^{1}$, Benzi M. Kluger ${ }^{1}$
}

${ }^{1}$ Department of Neurology, University of Colorado School of Medicine, CO 80045, USA; ${ }^{2}$ Division of Neurology, Department of Medicine, University of Alberta, Edmonton, T6G 2G3, Canada; ${ }^{3}$ Department of Neurology, University of California San Francisco Medical Center, San Francisco, CA 94122, USA; ${ }^{4}$ Department of Medicine, Emory University, Atlanta, GA, 30322, USA

Contributions: (I) Conception and design: JM Miyasaki, SH Sillau, BM Kluger; (II) Administrative support: JM Miyasaki, BM Kluger; (III) Provision of study materials or patients: JM Miyasaki, M Katz, N Galifianakis, BM Kluger; (IV) Collection and assembly of data: JM Miyasaki, CE Koljack, ZA Macchi, M Katz, N Galifianakis, LP Prizer, BM Kluger; (V) Data analysis and interpretation: JM Miyasaki, CE Koljack, ZA Macchi, SH Sillau, BM Kluger; (VI) Manuscript writing: All authors; (VII) Final approval of manuscript: All authors.

\#These authors contributed equally to this work.

Correspondence to: Benzi Kluger, MD, FAAN. Department of Neurology, University of Colorado, Academic Office 1, Mail Stop B-185, 12631 East 17th Avenue, Aurora, CO 80045, USA. Email: benzi.kluger@cuanschutz.edu.

Background: Parkinson's disease (PD) is a neurodegenerative disorder associated with caregiver burden. Higher rates of burden are associated with adverse outcomes for caregivers and patients. Our aim was to understand patient and caregiver predictors of caregiver burden in PD from a palliative care approach.

Methods: We conducted a cross-sectional analysis of baseline data from PD patients and caregivers in a randomized trial of outpatient palliative care at three study sites: University of Colorado, University of Alberta, and University of California San Francisco. The primary outcome measure of caregiver burden, the Zarit Burden Interview (ZBI), was compared against the following patient and caregiver variables: site of care, age, disease/caretaking duration, presence of atypical parkinsonism, race, income, education level, deep brain stimulation status, the Unified Parkinson's Disease Rating Scale (UPDRS) and Edmonton Symptom Assessment System Revised: Parkinson Disease (ESAS) for symptom severity and burden, the Montreal Cognitive Assessment (MoCA) for cognitive function, Quality of Life in Alzheimer's Disease (QOL-AD) scale for patient and caregiver perspectives on patient general quality of life, Parkinson's Disease Questionnaire 39 (PDQ-39) scale for health-related quality of life, Hospital Anxiety and Depression Scale (HADS) for patient and caregiver mood, Prolonged Grief Questionnaire, Functional Assessment of Chronic Illness TherapySpiritual Well-Being (FACIT-SP) of patient and caregiver, and Palliative Performance Scale for functional status. A stepwise multivariate linear regression model was used to determine associations with ZBI.

Results: A total of 175 patients $(70.9 \%$ male; average age $70.7 \pm 8.1$ years; average disease duration $117.2 \pm 82.6$ months), and 175 caregivers $(73.1 \%$ female; average age $66.1 \pm 11.1$ years) were included. Patient spiritual well-being (FACIT-SP Faith subscale, $\mathrm{r}^{2}=0.024, \mathrm{P}=0.0380$ ), patient health-related quality of life (PDQ-39, $\mathrm{r}^{2}=0.161, \mathrm{P}<0.0001$ ), caregiver depression (HADS Depression, $\mathrm{r}^{2}=0.062, \mathrm{P}=0.0014$ ), caregiver anxiety (HADS Anxiety, $\mathrm{r}^{2}=0.077, \mathrm{P}=0.0002$ ), and caregiver perspective on patient quality of life (QOL-AD Caregiver Perspective, $\mathrm{r}^{2}=0.088, \mathrm{P}<0.0001$ ) were significant contributors to $\mathrm{ZBI}$ scores.

Conclusions: Patient and caregiver factors contribute to caregiver burden in persons living with PD. These results suggest targets for future interventions to improve caregiver support.

Keywords: Caregivers; palliative care; Parkinson's disease

Submitted Sep 15, 2019. Accepted for publication Oct 02, 2019.

doi: 10.21037/apm.2019.10.01

View this article at: http://dx.doi.org/10.21037/apm.2019.10.01 


\section{Introduction}

Parkinson's disease (PD) is characterized by progressive motor deterioration and accumulation of non-motor symptoms including cognitive, psychiatric, and behavioral disturbances (1). Symptom burden for patients with PD is similar to those with metastatic cancer (2). Informal caregivers provide integral care and support to PD patients in the community, and their sacrifices for patient care add to PD's societal cost. This cost can include loss of working and leisure time, financial hardship, and increased morbidity (3). Caregiver burden is defined as the perception of strain and stress resulting from a perceived obligation to provide care for their loved one with PD (4). The level of burden for caring for patients with $\mathrm{PD}$ is comparable to caregivers for stroke patients (5). Notably, higher rates of burden also lead to increased caregiver morbidity which in turn impacts PD patient quality of life $(6,7)$. Understanding the factors which contribute to caregiver burden is paramount to developing strategies that support caregivers.

Studies have shown that several patient characteristics in PD contribute to higher rates of caregiver burden with the greatest predictors being disease severity and the presence of non-motor symptoms, especially neuropsychiatric disturbances (8-10). Additionally, worse self-reported quality of life, and higher rates of self-reported depression in PD patients lead to higher caregiver burden $(6,7)$. Caregiver characteristics such as psychological status, quality of life, anticipatory and illness-associated grief are associated with increased burden $(7,9,11,12)$. In these studies, patient characteristics and caregiver burden, disease severity, symptomology, caregiver grief, psychological state of both patient and caregiver, and quality of life were shown to influence caregiver burden in PD.

Palliative care is a relatively new approach for supporting both persons living with PD and their families (13). While prior studies of caregiver burden provide important predictors, they did not include important palliative care domains such as spiritual well-being, grief, overall symptom burden, or burdens associated with receiving medical care (e.g., hospitalizations). They also did not separately include caregiver perspectives on patient quality of life which may differ substantially from the perceptions of patients (14). Evaluating caregiver burden in a comprehensive manner is necessary to inform future efforts to improve caregiver support and reduce burden through palliative care approaches. In other causes of dementia, there is an inverse association between caregiver spirituality and burden among caregivers suggesting that a similar trend could be seen in PD (15). The aim of this study to assess patient and caregiver characteristics, including spirituality and grief, which affect caregiver burden in a palliative PD population with known moderate to high palliative care needs.

\section{Methods}

We performed a cross-sectional study using baseline data from a randomized controlled comparative effectiveness trial of outpatient palliative care versus standard care for individuals with PD and other forms of parkinsonism performed at three academic medical centers (16). Institutional review board approval was obtained at each of the participating sites which included the University of Colorado Anschutz Medical Campus (CU), University of Alberta (University of Alberta), and University of California San Francisco (UCSF) (ClinicalTrials.gov NCT 02533921). Participants were recruited from specialized movement disorder programs, community neurology practices, patient support groups, and the Michael J. Fox Foundation trial finder. Investigators used the University of California San Diego Brief Assessment of Capacity to Consent (UBACC) to assess individuals for capacity prior to enrollment (17). If participants lacked capacity for consent, their legally authorized medical proxy provided consent with participant assent. Baseline data was obtained from enrolled patients and caregivers, and included outcome measures relating to motor symptoms, cognitive complaints, functional impairment, mood disturbances, quality of life, patient grief, caregiver burden, and palliative performance. Study data was collected and managed using REDCap (Research Electronic Data Capture) electronic data capture tools hosted at the Colorado Clinical \& Translational Sciences Institute (CCTSI) $(18,19)$.

\section{Participants}

Eligibility for enrollment included: English speaking fluency, age $>40$ years, and diagnosis of probable PD using the UK Parkinson's Disease Society Brain Bank criteria (20), or a diagnosis of an another neurodegenerative parkinsonian condition: progressive supranuclear palsy (PSP), corticobasal degeneration (CBD), multiple systems atrophy (MSA), or Lewy Body Dementia (LBD). Participants also required high to moderate palliative care needs based on the Palliative Care Needs Assessment Tool (PC-NAT) modified for PD (16). Caregivers were 
identified by the patient with the question: "Could you please tell us the one person who helps you the most with your PD outside of clinic?" or through caregiver selfreferral. One caregiver was enrolled for each participant when available. Participants were excluded based on the following factors: (I) immediate and urgent palliative care needs (these patients were not randomized into the larger trial and were offered appropriate services immediately); (II) unable or unwilling to commit to study procedures; (III) presence of additional chronic medical illnesses which may require palliative services (e.g., metastatic cancer); or (IV) a history of palliative care treatment and/or hospice services. After enrollment, demographic information from both patients and caregivers was collected including: age, disease duration, caregiving role duration, gender, race/ethnicity, education level, marital status, income and deep brain stimulation (DBS) status.

\section{Outcome measures}

The primary outcome measure was the Zarit Burden Interview (ZBI), a 12-item scale with responses scored on a 5 -point Likert scale from 0 (never) to 4 (nearly always) (21). A ZBI summary score ranged from 0 to 48 with higher scores indicating higher levels of caregiver burden.

\section{Patient predictor variables}

Patient predictor variables included age, disease duration, presence of atypical parkinsonism, the Unified Parkinson's Disease Rating Scale (UPDRS) to monitor disease progression (22), the Edmonton Symptom Assessment System Revised: Parkinson Disease (ESAS-PD) to measure symptom burden (2), and the Montreal Cognitive Assessment (MoCA) to measure cognitive function (23). General patient quality of life was measured using the Quality of Life in Alzheimer's Disease (QOL-AD) scale (24). Health-related quality of life (HRQoL) was measured with the Parkinson's Disease Questionnaire 39 (PDQ-39) (25). Patient mood was measured by the Hospital Anxiety and Depression Scale (HADS) and patient grief by the Prolonged Grief Questionnaire (PG12) (26,27). Overall functional status was measured using the Palliative Performance Scale (PPS) (28). Spiritual well-being of patients was assessed using the Functional Assessment of Chronic Illness Therapy-Spiritual Well-Being (FACIT-SP), a 12-item scale comprised of three subscales: meaning, peace, and faith (29). We also included rates of patient healthcare utilization including emergency room visits, inpatient hospitalizations, home health services, social work, psychotherapy, and religious/spiritual counseling services.

\section{Caregiver predictor variables}

Caregiver predictor variables included study site, duration of caregiving, shared living situation relative to patient, age, sex, race, marital status, education, income, caregiverperceived patient quality of life (QOL-AD Caregiver Reported) (24), and caregiver healthcare utilization including emergency room visits, inpatient hospitalizations and services related to social work, psychotherapy, or religious/spiritual counseling. Caregiver mood was assessed using the HADS (26) and spiritual well-being of caregivers was assessed using the FACIT-SP (29).

\section{Statistical analysis}

Univariate relationships between ZBI and explanatory variables were assessed with correlation statistics for continuous variables and scales, as well T-test or ANOVA type models for categorical variables. Multivariate models were fit for ZBI using multiple regression. Model selection algorithms, including backwards elimination and stepwise guided the construction of final multivariate models. The effects of each explanatory variable were presented as partial $\mathrm{R}$-squares, adjusted for the presence of the other variables in the model. Partial R-squares were obtained by taking $\mathrm{Z}$ scores of all the variables in the model, which standardized the parameter estimates to correlation coefficients ( $r$ ).

\section{Results}

\section{Participant characteristics}

We recruited 175 patients $(70.9 \%$ male; average age of $70.7 \pm 8.1$ years; average disease duration of $117.2 \pm$ 82.6 months) and 175 caregivers (73.1\% female; average age of $66.1 \pm 11.1$ years). Average duration of caregiving was 68.5 months ( $\mathrm{SD}=62.8$ ). In $90.9 \%$ of cases the patient and caregiver shared the same household, and in $81.7 \%$ of cases the patient and caregiver were spouses. More than $90 \%$ of both patients and caregivers identified as white, and more than 90\% identified as non-Hispanic. Table 1 details the complete demographic information for patients and caregivers.

\section{Patient characteristics associated with caregiver burden}

Univariate models of patient characteristics showed 
Table 1 Patient \& caregiver characteristics

\begin{tabular}{|c|c|c|}
\hline Variables & Patients & Caregivers \\
\hline Age & $70.7 \pm 8.1$ & $66.1 \pm 11$ \\
\hline $\begin{array}{l}\text { Disease/caregiving duration } \\
\text { (months) }\end{array}$ & $117.2 \pm 82.6$ & $68.5 \pm 62.8$ \\
\hline MoCA & $23.4 \pm 5.1$ & N/A \\
\hline MDS UPDRS (motor) & $42.0 \pm 18.9$ & N/A \\
\hline HADS Anxiety & $7.4 \pm 4.0$ & $7.4 \pm 3.6$ \\
\hline HADS Depression & $7.1 \pm 3.7$ & $4.3 \pm 3.1$ \\
\hline PG12 & $24.4 \pm 5.1$ & $\mathrm{~N} / \mathrm{A}$ \\
\hline QOL AD & $34.7 \pm 5.6$ & $33.8 \pm 6.0$ \\
\hline PDQ-39 & $58.1 \pm 28.4$ & N/A \\
\hline FACIT-SP & $28.6 \pm 9.2$ & $33.5 \pm 8.7$ \\
\hline ZBI & $\mathrm{N} / \mathrm{A}$ & $17.4 \pm 7.9$ \\
\hline Shares Household with patient & N/A & $159(90.9)$ \\
\hline Sex (male) & $124(70.9)$ & $47(26.9)$ \\
\hline Atypical PD & $25(14.3)$ & N/A \\
\hline \multicolumn{3}{|l|}{ Race/ethnicity } \\
\hline African American & $2(1.1)$ & $1(0.6)$ \\
\hline Asian & $5(2.9)$ & $8(4.6)$ \\
\hline Native American & $1(0.6)$ & $1(0.6)$ \\
\hline White & $160(91.4)$ & $159(90.9)$ \\
\hline Multiracial & $2(1.1)$ & $2(1.1)$ \\
\hline Other & $4(2.3)$ & $3(1.7)$ \\
\hline No response & $0(0.0)$ & $1(0.6)$ \\
\hline \multicolumn{3}{|l|}{ Education } \\
\hline Less than high school diploma & $12(6.9)$ & $9(5.1)$ \\
\hline High school diploma & $12(6.9)$ & $14(8.0)$ \\
\hline Some college & $24(13.7)$ & $18(10.3)$ \\
\hline $\begin{array}{l}\text { Associate's or Bachelor's } \\
\text { degree }\end{array}$ & $47(26.9)$ & $65(37.1)$ \\
\hline Post-graduate degree & $79(45.1)$ & $68(38.9)$ \\
\hline \multicolumn{3}{|l|}{ Marital status } \\
\hline Never married & $4(2.3)$ & $5(2.9)$ \\
\hline Married & $151(86.3)$ & $157(89.7)$ \\
\hline Widowed, separated or divorced & $19(10.9)$ & $11(6.3)$ \\
\hline Unknown & $1(0.6)$ & $2(1.1)$ \\
\hline
\end{tabular}

Table 1 (continued)
Table 1 (continued)

\begin{tabular}{lcc}
\hline Variables & Patients & Caregivers \\
\hline Income & $16(9.1)$ & $9(5.1)$ \\
$\$ 0-29,999$ & $24(13.7)$ & $23(13.1)$ \\
$\$ 30,000-59,999$ & $26(14.9)$ & $26(14.9)$ \\
$\$ 60,000-99,999$ & $51(29.1)$ & $58(33.1)$ \\
$\$ 100,000+$ & $16(9.1)$ & $16(9.1)$ \\
Unknown & & \\
Healthcare services utilization & $27(15.4)$ & $8(4.6)$ \\
Emergency room & $10(5.7)$ & $5(2.9)$ \\
Inpatient hospitalization & $27(15.4)$ & $0(0.0)$ \\
Home health & $41(24.7)$ & $23(13.1)$ \\
$\begin{array}{l}\text { Social work, psychotherapy, or } \\
\text { religious/spiritual counseling }\end{array}$ & & \\
DBS surgery status (yes) & $28(16.0)$ & $\mathrm{N} / \mathrm{A}$
\end{tabular}

Data are shown as number (\%) or mean \pm standard deviation. MoCA, Montreal Cognitive Assessment; MDS UPDRS, Movement Disorder Society Unified Parkinson's Disease Rating Scale; HADS, Hospital Anxiety and Depression Scale; PG12, Prolonged Grief questionnaire; QOL AD, Quality of Life in Alzheimer's Disease scale; PDQ-39, Parkinson's Disease Questionnaire 39; FACIT-SP, Functional Assessment of Chronic Illness Therapy-Spiritual Well-being scale; ZBI, Zarit Burden Interview; DBS, deep brain stimulation; N/A, not applicable.

significant patient predictors of ZBI were all patient FACIT-SP subscores, HADS Anxiety and Depression, PDQ-39, QOL-AD, the presence of atypical parkinsonism, ESAS, MDS UPDRS (motor subscale), PPS, number of medications, emergency room evaluations, inpatient hospitalization, and receiving home health services. Stepwise linear regression models had an overall fit of $F(3,137)=35.9, \mathrm{r}^{2}=0.5023(\mathrm{P}<0.0001)$ and with backwards elimination the FACIT-SP Faith subscale $\left(r^{2}=0.024\right.$, $\mathrm{P}=0.0380)$ and PDQ-39 $\left(\mathrm{r}^{2}=0.161, \mathrm{P}<0.0001\right)$ remained significantly associated with ZBI. Details for univariate and multivariate correlations between ZBI and patient predictor variables are detailed in Table 2.

\section{Caregiver characteristics associated with caregiver burden}

Univariate models of caregiver characteristics showed 
Table 2 Patient outcome variables contributing to ZBI

\begin{tabular}{|c|c|c|}
\hline Variables in model & Pearson $r$ and $P$ value & $\begin{array}{l}\text { Significant results from backwards } \\
\text { elimination linear regression model }\end{array}$ \\
\hline \multicolumn{3}{|l|}{ Spiritual well-being } \\
\hline FACIT-SP peace items & $r=-0.29, P<0.0001$ & \\
\hline FACIT-SP faith items & $r=-0.25, P<0.0001$ & $r^{2}=0.024, P=0.0380$ \\
\hline HADS anxiety-patient & $r=0.34, P<0.0001$ & \\
\hline HADS depression-patient & $r=0.35, P<0.0001$ & \\
\hline \multicolumn{3}{|l|}{ Patient quality of life } \\
\hline Atypical PD & $r=0.19, P=0.0138$ & \\
\hline ESAS-PD & $r=-0.31, P<0.0001$ & \\
\hline MDS UPDRS-3 & $r=0.20, P=0.0091$ & \\
\hline MOCA & $r=-0.20, P=0.0114$ & \\
\hline \multicolumn{3}{|l|}{ Functional status } \\
\hline Palliative performance scale & $r=-0.23, P=0.0023$ & \\
\hline \multicolumn{3}{|c|}{ Medications \& healthcare utilization } \\
\hline
\end{tabular}

Overall model fit: $F(2,150)=20.4, r^{2}=0.2142, P<0.0001$. ZBI, Zarit Burden Interview; FACIT-SP, Functional Assessment of Chronic Illness Therapy-Spiritual Well-being scale; HADS, Hospital Anxiety and Depression Scale; QOL, Quality of Life; QOL AD, Quality of Life in Alzheimer's Disease scale.

significant caregiver predictors of ZBI were study site, caregiver age, caregiving duration, caregiver FACIT-SP meaning and peace sub-scores but not faith, HADS anxiety and depression, and caregiver-reported patient QOL-AD. Stepwise linear regression models had an overall model fit of $F(5,141)=26.7, \mathrm{r}^{2}=0.4865(\mathrm{P}<0.0001)$. There was a significant contribution of study site $\left(\mathrm{r}^{2}=0.098, \mathrm{P}=0.0007\right)$, caregiver anxiety (HADS Anxiety Caregiver score, $\mathrm{r}^{2}=0.077$, $\mathrm{P}=0.0002$ ), caregiver depression (HADS Depression Caregiver score, $\left.\mathrm{r}^{2}=0.062, \mathrm{P}=0.0014\right)$ and caregiver reported patient quality of life (QOL AD-Caregiver Reported score, $\left.\mathrm{r}^{2}=0.088, \mathrm{P}<0.0001\right)$ to caregiver burden $(\mathrm{ZBI})$. Details for univariate and multivariate correlations between ZBI and caregiver predictor variables are detailed in Table 3.

Of note, while the mean 12-item ZBI score for this caregiver sample was $17.4(\mathrm{SD}=7.9)$, there was a significant difference in ZBI by study site after adjusting for caregiver gender, caregiver age, MOCA score, duration of caregiving, and presence of atypical PD, $F(2,82.6)=7.4, \mathrm{P}<0.0011$. ZBI at the CU site (mean $=20.1, \mathrm{SD}=7.4$ ) was significantly higher than at University of Alberta (mean $=14.5, \mathrm{SD}=8.1)(\mathrm{P}=0.0008)$. There was no significant difference found between $\mathrm{CU}$ and UCSF $(\mathrm{P}=0.14)$ or UCSF and University of Alberta $(\mathrm{P}=0.11)$. Adjusted ZBI scores by site are found in Table 4 . 
Table 3 Caregiver outcome variables contributing to ZBI

\begin{tabular}{|c|c|c|}
\hline Variables used in model & Pearson $r$ and $P$ value & $\begin{array}{l}\text { Significant results from backwards } \\
\text { elimination linear regression model }\end{array}$ \\
\hline Site & $r=0.24, P=0.0005$ & $r^{2}=0.098, P=0.0007$ \\
\hline Age & $r=-0.21, P=0.0079$ & \\
\hline \multicolumn{3}{|l|}{ Spiritual well-being } \\
\hline FACIT-SP peace items & $r=-0.48, P<0.0001$ & \\
\hline \multicolumn{3}{|l|}{ Mood } \\
\hline HADS anxiety-caregiver & $r=0.48, P<0.0001$ & $r^{2}=0.077, P=0.0002$ \\
\hline
\end{tabular}

Overall model fit: $F(5,141)=26.7, r^{2}=0.4865, \mathrm{P}<0.0001$. Zarit Burden Interview; FACIT-SP, Functional Assessment of Chronic IIIness Therapy-Spiritual Well-being scale; HADS, Hospital Anxiety and Depression Scale; PDQ-39, Parkinson's Disease Questionnaire 39; QOL AD, Quality of Life in Alzheimer's Disease scale; ESAS-PD, Edmonton Symptom Assessment System Revised: Parkinson Disease; MDS UPDRS-3, Movement Disorder Society Unified Parkinson's Disease Rating Scale (Motor Items); MOCA, Montreal Cognitive Assessment.

Table 4 Adjusted difference in Zarit Burden Interview score by study site

\begin{tabular}{llll}
\hline \multirow{2}{*}{ Study site } & \multicolumn{3}{l}{ Zarit Burden Interview score } \\
\cline { 2 - 4 } & Mean & SD & SE \\
\hline University of Alberta & 13.72 & 7.99 & 1.31 \\
$\begin{array}{l}\text { University of Colorado Anschutz } \\
\text { Medical Campus }\end{array}$ & 19.64 & 6.70 & 0.96 \\
$\begin{array}{l}\text { University of California San } \\
\text { Francisco }\end{array}$ & 16.83 & 7.04 & 1.00 \\
\hline
\end{tabular}

\section{Conclusions}

We found that patient spiritual well-being, HRQoL, caregiver anxiety, caregiver depression and caregiver perceptions of patient quality of life were significantly associated with caregiver burden in a population of persons living with PD with moderate to high palliative care needs. We also found significant differences in caregiver burden across geographic sites. While several other candidate predictors were found to be significant in univariate models, these predictors were not significant in our final multivariate models suggesting that their associations with caregiver burden were smaller and may be partially mediated by other variables. Of note, these predictors may still be important for individual patients. Strengths of this study include it being a large, multi-site study evaluating a large pool of candidate variables in a population reflective of outpatient palliative care clinics. Overall, these results suggest a comprehensive approach to both patient and caregiver needs with a particular focus on patient function and caregiver mood may be useful in addressing caregiver burden.

\section{Patient health-related quality of life \& faith}

Patient HRQoL and faith were significant predictors of caregiver burden. Our measure of HRQoL, the PDQ-39, is the most frequently used disease-specific measure of $\mathrm{PD}$ health status and assesses the impact of disease on specific areas of functioning and well-being. It is largely driven by patent perceptions of activities of daily living, cognition, depression, functional mobility, and quality of life, as well as social relationships, communication and support (25). Its association with caregiver burden could reflect increasing caregiver support needed for persons with limited function, diminished support from their primary relationship for persons with communication disabilities and diminished community and family social support $(30,31)$. The finding of faith may reflect the role of interpersonal and spiritual/ religious support systems as a means of coping with PD. 
Studies show that aside from family, the greatest source of social support is related to spiritual or religious communities and perceptions of spiritual well-being are connected to health-related quality of life $(29,32)$. Our findings are in line with of previous studies which demonstrated associations between social support and religious or spiritual institutions (33-35). Resources and support from religious or spiritual communities might be targets for improving the lives of both patients with PD and their caregivers.

\section{Caregiver mood}

Similar to previous investigations, we found caregiver burden was associated with caregiver depression and anxiety $(7,9,11,12)$. This reinforces the notion that the stresses of caretaking confer significant emotional duress onto the caregiver but may also reflect the impact of mood on perceptions of burden and coping abilities. Interventions which aim to alleviate caregiver mood disturbances should also be considered in the PD population as part of comprehensive palliative care. Although measures of caregiver spiritual well-being were not significantly associated with burden in our multivariate model, they were univariate predictors and the regular inclusion of caregiver spiritual counseling or pastoral care might also mitigate the psychologic effects of burden on PD caregivers.

\section{Caregiver perspective on patient quality of life}

Caregiver perceptions of patient quality of life were also predictors of caregiver burden. Prior studies of caregiver and patient perspectives on patient quality of life in PD demonstrate notable differences and suggest that patient and caregiver reports are not interchangeable and reflect different priorities and perceptions of illness burden $(14,36)$. There are several potential explanations for this finding which are not mutually exclusive. First, caregiver perceptions of patient quality of life would be expected to more closely reflect the caregiver experience and may be more largely based on symptoms that are bothersome and noticeable to the caregiver but carry less weight for patients. Second, caregivers may be more accurate in assessing certain symptoms and aspects of quality of life due to changes in patient awareness with cognitive dysfunction of under-reporting due to perceived stigma. Lastly, caregivers with higher burden may be more prone to higher reporting of dysfunction as a means of calling attention to difficulties in their living situation or from reduced coping resources. Regardless of the cause of this association, these results support a need to assess both patient and caregiver perceptions of patient quality of life, symptoms and function as distinct data points in creating plans of care for patient-caregiver dyads.

\section{Study site differences}

There were significant differences in caregiver burden between study sites. The University of Alberta site's caregivers reported less caregiver burden at baseline despite otherwise similar patient and caregiver characteristics. One possible factor contributing to this difference is the availability of government paid home-based healthcare services in the Canadian patient population compared to those in the United States. Home healthcare-based palliative services through the United States Medicaid program are limited, more complete home palliative care support is only consistently available through hospice and is limited to patients with a life expectancy of 6 months or less. Unfortunately, PD patients may have very low functional abilities and high palliative care needs for much longer than their last 6 months of life (37). Other possible site characteristics contributing to the difference in caregiver burden include differences in clinical care, services offered and received by participants prior to receiving the palliative care intervention, or local healthcare policies as Medicaid home health benefits are determined by state as well as federal laws. Finally, it is possible that site differences could reflect differences in referral and enrollment patterns as the palliative care needs assessment tool used for this study allowed for moderate to high needs in patients and/or caregivers.

\section{Other findings}

Although not significant in our final multivariate model, univariate results suggest patient and caregiver demographics and patient healthcare utilization in the form of emergency room visits, hospitalizations and home health services could contribute to caregiver burden for individual caregivers affected by these issues. Possible explanations for healthcare utilization as a contributor to caregiver burden include duress related to witnessing a loved one's acute illness, prognostic uncertainty, and/or increasing demands placed on caregivers during recovery following hospital discharge. Home health services are 
typically utilized in later stages of the disease and are likely reflective of patient functional decline as well as caregiver needs for outside support. Other patient factors with univariate associations with higher ZBI were cognitive impairment, motor symptoms, depression, anxiety, overall symptom burden, global function and these factors have been reported in other studies (9-11). It is possible that the lack of an association in our multivariate model is that the functional impact of these symptoms was better captured in the PDQ-39. An unexpected finding in our cohort was that higher caregiver age was associated with reduced caregiver burden. This may be attributable to changes in patient care expectations for both caregiver and patient, the relinquishment of caregiver career obligations with retirement, or better ability of adult children to share in caregiving responsibilities. Additional research using qualitative methods could better explore these contributors and inform future studies on caregiver burden interventions for application in both outpatient and inpatient settings.

\section{Limitations}

While we are unable to derive causal conclusions from our sample due to the its cross-sectional design, our study provides insights into which caregivers are at higher risk for caregiver burden. However, these associations can inform future primary and specialized palliative programs to reduce caregiver burden in PD patient populations, as well as future research studies. While relatively large, our sample size still precludes finding significance for variables with small but possibly clinically significant association. Similarly, our approach to model building could influence our conclusions and our use of linear regression may underestimate the impact of predictors with nonlinear relationships with our primary outcome. Our study cohort was largely white, educated and as participants in a palliative care study may not be representative of PD patients in general. Our conclusions are also affected by how our outcome measures operationalize key constructs, and this is particularly relevant for our primary outcome, the ZBI. Notably, burden is only one dimension of the total caregiving experience and the focus on the negative aspects of caregiving may neglect equally important positive aspects of caregiving such as the potential for growth and self-efficacy (38). This is particularly relevant to a comprehensive palliative care approach as clinical support for caregivers is also often overly focused on sources of suffering and neglects systematically exploring existing sources of joy and meaning or opportunities for growth.

\section{Future directions}

Our findings add to prior cross-sectional studies on the association of patient and caregiver factors with caregiver burden. Longitudinal studies are needed to determine the clinical value of these associations in predicting burnout. Similarly, interventional studies are needed to determine the therapeutic value of targeting those variables that are modifiable in reducing burden and preventing burnout. Given the lack of diversity in our study cohort, efforts should also be made to study these issues in more diverse populations. Our finding of differences across study sites suggests an important avenue of future epidemiologic research to compare caregiver wellbeing and burden across national and international healthcare systems as a means of determining which models of care best support family caregivers.

Both patient and caregiver characteristics contribute to caregiver burden in persons living with $\mathrm{PD}$. This includes patient perceptions of HRQoL, patient spiritual wellbeing, caregiver anxiety, caregiver depression, and caregiver perceptions of patient quality of life. Providers should make holistic assessments of the patient-caregiver unit when addressing caregiver burden in palliative PD populations. Targets for future interventions include identifying social support systems and allocation of resources to improve caretaking support and caregiver burden in succession. Comprehensive patient symptom management, caregiver psychological support, and techniques to address the caregiver's perspective on patient quality of life (e.g., group spiritual counseling for the patient and caregiver) have the potential to improve caregiver burden.

\section{Acknowledgments}

Funding: Research reported in this publication (or work or article) was (partially) funded through a Patient-Centered Outcomes Research Institute (PCORI) Project Program Award IHS-1408-20134.

\section{Footnote}

Conflicts of Interest: The authors have no conflicts of interest to declare.

Ethical Statement: The authors are accountable for all 
aspects of the work in ensuring that questions related to the accuracy or integrity of any part of the work are appropriately investigated and resolved. This study was approved by the Colorado Multiple Institutional Review Board (COMIRB) at the University of Colorado Denver (No.: 15-0814). All participants gave informed consent before taking part in this study. A copy of the written consent is available for review by the Editor-in-Chief of this journal.

Disclaimer: The views, statements, opinions in this publication are solely the responsibility of the authors and do not necessarily represent the views of the PatientCentered Outcomes Research Institute (PCORI), its Board of Governors or Methodology Committee and the Development and Informatics Service Center (DISC) (NIH/NCRR Colorado CTSI Grant \# UL1 RR025780). All tables included in this study are original and have not been previously published nor appeared in copyright form elsewhere.

\section{References}

1. Jankovic J. Parkinson's disease: clinical features and diagnosis. J Neurol Neurosurg Psychiatry 2008;79:368-76.

2. Miyasaki JM, Long J, Mancini D, et al. Palliative care for advanced Parkinson disease: an interdisciplinary clinic and new scale, the ESAS-PD. Parkinsonism Relat Disord 2012;18:S6-9.

3. Mateus C, Coloma J. Health economics and cost of illness in Parkinson's disease. Eur Neurol Rev 2013;8:6-9.

4. Edwards NE, Ruettiger KM. The influence of caregiver burden on patients' management of Parkinson's disease: implications for rehabilitation nursing. Rehabil Nurs 2002;27:182-6.

5. Kim KS, Kim BJ, Kim KH, et al. Subjective and objective caregiver burden in Parkinson's disease. Taehan Kanho Hakhoe Chi 2007;37:242-8.

6. Peters M, Fitzpatrick R, Doll H, et al. Does self-reported well-being of patients with Parkinson's disease influence caregiver strain and quality of life? Parkinsonism Relat Disord 2011;17:348-52.

7. Caap-Ahlgren M, Dehlin O. Factors of importance to the caregiver burden experienced by family caregivers of Parkinson's disease patients. Aging Clin Exp Res 2002;14:371-7.

8. Kudlicka A, Clare L, Hindle JV. Quality of life, health status and caregiver burden in Parkinson's disease: relationship to executive functioning. Int J Geriatr Psychiatry 2014;29:68-76.

9. Martínez-Martín P, Forjaz MJ, Frades-Payo B, et al. Caregiver burden in Parkinson's disease. Movement Disord 2007;22:924-31.

10. Martinez-Martin P, Rodriguez-Blazquez C, Forjaz MJ, et al. Neuropsychiatric symptoms and caregiver's burden in Parkinson's disease. Parkinsonism Relat Disord 2015;21:629-34.

11. Holley CK, Mast BT. The impact of anticipatory grief on caregiver burden in dementia caregivers. Gerontologist 2009;49:388-96.

12. Carter JH, Lyons KS, Lindauer A, et al. Pre-death grief in Parkinson's caregivers: a pilot survey-based study. Parkinsonism Relat Disord 2012;18:S15-8.

13. Miyasaki JM, Kluger B. Palliative care for Parkinson's disease: has the time come? Curr Neurol Neurosci Rep 2015;15:26.

14. Sebring K, Shattuck J, Berk J, et al. Assessing the validity of proxy caregiver reporting for potential palliative care outcome measures in Parkinson's disease. Palliat Med 2018;32:1522-8.

15. McLennon SM, Habermann B, Rice M. Finding meaning as a mediator of burden on the health of caregivers of spouses with dementia. Aging Ment Health 2011;15:522-30.

16. Kluger BM, Katz M, Galifianakis N, et al. Does outpatient palliative care improve patient-centered outcomes in Parkinson's disease: Rationale, design, and implementation of a pragmatic comparative effectiveness trial. Contemp Clin Trials 2019;79:28-36.

17. Jeste DV, Palmer BW, Appelbaum PS, et al. A new brief instrument for assessing decisional capacity for clinical research. Arch Gen Psychiat 2007;64:966-74.

18. Harris PA, Taylor R, Thielke R, et al. Research electronic data capture (REDCap)--a metadata-driven methodology and workflow process for providing translational research informatics support. J Biomed Inform 2009;42:377-81.

19. Harris PA, Taylor R, Minor BL, et al. The REDCap consortium: Building an international community of software platform partners. J Biomed Inform 2019;95:103208.

20. Hughes AJ, Ben-Shlomo Y, Daniel SE, et al. What features improve the accuracy of clinical diagnosis in Parkinson's disease: a clinicopathologic study. Neurology 1992;42:1142-6.

21. Hébert R, Bravo G, Préville M. Reliability, validity and reference values of the Zarit Burden Interview for assessing 
informal caregivers of community-dwelling older persons with dementia. Can J Aging 2000;19:494-507.

22. Goetz CG, Tilley BC, Shaftman SR, et al. Movement Disorder Society-sponsored revision of the Unified Parkinson's Disease Rating Scale (MDS-UPDRS): Scale presentation and clinimetric testing results. Movement Disord 2008;23:2129-70.

23. Dalrymple-Alford JC, MacAskill MR, Nakas CT, et al. The MoCA well-suited screen for cognitive impairment in Parkinson disease. Neurology 2010;75:1717-25.

24. Thorgrimsen L, Selwood A, Spector A, et al. Whose quality of life is it anyway?: The validity and reliability of the Quality of Life-Alzheimer's Disease (QoL-AD) scale. Alzheimer Dis Assoc Disord 2003;17:201-8.

25. Jenkinson C, Fitzpatrick R, Peto V, et al. The Parkinson's Disease Questionnaire (PDQ-39): development and validation of a Parkinson's disease summary index score. Age Ageing 1997;26:353-7.

26. Zigmond AS, Snaith RP. The hospital anxiety and depression scale. Acta Psychiat Scand 1983;67:361-70.

27. Prigerson HG, Horowitz MJ, Jacobs SC, et al. Prolonged grief disorder: Psychometric validation of criteria proposed for DSM-V and ICD-11. PLoS Med 2009;6:e1000121.

28. Morita T, Tsunoda J, Inoue S, et al. Validity of the palliative performance scale from a survival perspective. J Pain Symptom Manage 1999;18:2-3.

29. Peterman AH, Fitchett G, Brady MJ, et al. Measuring spiritual well-being in people with cancer: the functional assessment of chronic illness therapy-Spiritual Wellbeing Scale (FACIT-Sp). Ann Behav Med 2002;24:49-58.

Cite this article as: Macchi ZA, Koljack CE, Miyasaki JM, Katz M, Galifianakis N, Prizer LP, Sillau SH, Kluger BM. Patient and caregiver characteristics associated with caregiver burden in Parkinson's disease: a palliative care approach. Ann Palliat Med 2020;9(Suppl 1):S24-S33. doi: 10.21037/ apm.2019.10.01
30. Shiba K, Kondo N, Kondo K. Informal and Formal Social Support and Caregiver Burden: The AGES Caregiver Survey. J Epidemiol 2016;26:622-8.

31. Yang Z, Tian Y, Fan Y, et al. The mediating roles of caregiver social support and self-efficacy on caregiver burden in Parkinson's disease. J Affect Disord 2019;256:302-8.

32. Neill CM, Kahn AS. The Role of Personal Spirituality and Religious Social Activity on the Life Satisfaction of Older Widowed Women. Sex Roles 1999;40:319-29.

33. Ellison CG, George LK. Religious Involvement, Social Ties, and Social Support in a Southeastern Community. J Sci Stud Relig 1994;33:46-61.

34. van Olphen J, Schulz A, Israel B, et al. Religious involvement, social support, and health among AfricanAmerican women on the east side of Detroit. J Gen Intern Med 2003;18:549-57.

35. Lim C, Putnam RD. Religion, Social Networks, and Life Satisfaction. Am Sociol Rev 2010;75:914-33.

36. Demeulemeester F, De Letter M, Miatton M, et al. Quality of life in patients with PD and their caregiving spouses: A view from both sides. Clin Neurol Neurosurg 2015;139:24-8.

37. Poewe $W$. The natural history of Parkinson's disease. J Neurol 2006;253:VII2-6.

38. Pakenham KI. The positive impact of multiple sclerosis (MS) on carers: associations between carer benefit finding and positive and negative adjustment domains. Disabil Rehabil 2005;27:985-97. 